\title{
AN ANGIOGRAPHIC TEST OF COLLATERAL CIRCULATION THROUGH THE ANTERIOR SEGMENT OF THE CIRCLE OF WILLIS
}

\author{
BY \\ C. B. SEDZIMIR \\ From the Regional Neurosurgical Centre, Liverpool
}

This communication is concerned with the angiographic estimation of collateral circulation through the anterior segment of the circle of Willis. The collateral circulation, which may be present between the ophthalmic artery and the branches of the external carotid artery and the posterior segment of the circle of Willis carrying blood from the vertebral circulation, is outside the scope of this discussion.

This angiographic test of the collateral circulation has been carried out sporadically in neurosurgical services all over the world. Personal experience of it extends over 13 years.

The estimation of its adequacy through the anterior cerebral arterial complex is of great practical importance to neurosurgeons if it is proposed to treat certain intracranial aneurysms by ligating the internal carotid artery or the anterior cerebral artery. In recent years general physicians and neurologists have been interested in its role, as spontaneous or traumatic thrombosis of the internal carotid artery has become a well established diagnosable entity. The many and variable clinical symptoms and signs of that condition (which is sometimes symptomless) can be largely explained by the anatomical and dynamic state of the collateral circulation through the anterior segment of the circle of Willis. Other possible collateral channels may also be brought into play later.

The stimulus to employ this angiographic test in a larger number of patients was provided by the observation that some elderly subjects possessed an excellent collateral circulation. Two questions were posed: (1) Can angiography substantiate or refute the belief that collateral circulation deteriorates with age so that thrombosis or ligation of the internal carotid artery becomes excessively hazardous after the age of 50 ? (2) To what extent can the collateral circulation be disturbed by other factors ?

In an attempt to elucidate the answer to these questions, the test was performed on patients subjected to diagnostic angiography in the Regional
Neurosurgical Centre at Liverpool from 1954 to 1957. At first digital compression was carried out on each case.

Unfortunately at the beginning of 1956 the two surgeons carrying out this experiment were warned that dangerous amounts of radiation were being received on their hands. In view of this the routine performance of the test was discontinued. Thus only 712 patients had the collateral circulation tested.

The view held here is that angiography is the safest method of investigation in cerebral contras radiography. Its technique and the reasons why if is considered safe have been discussed at lengtll $\vec{R}$ in a previous communication (Sedzimir, 1955). The $\frac{8}{0}$ series is now close to 2,000 angiograms withoug death, with one case of transient hemiparesis, tw\& transient supranuclear facial palsies, and one cas. $\vec{\theta}$ of infection of the neck which resolved without surgical intervention.

\section{Technique of Test}

All angiography was performed under general anaesthesia. Contrast medium was injected into one carotid artery just below the carotid sinus by the percutaneous method. A few seconds before the start of the injection, the opposite internal carotid artery was digitally compressed. Three exposures in the antero-posterior projection were made. Frequently a bilateral estimation was carried out at the same sitting.

\section{Definitions}

For the purpose of this experiment it was arbitrarily defined that collateral circulation through the anterior segment of the circle of Willis was classified as:

(1) "Good" if in addition to the ipsilateral arteries, the contralateral anterior and middle cerebral arteries were filled (Fig. 1).

(2) "Poor" if the contralateral anterior cerebral $D$ artery was filled, the contrast medium not entering the contralateral middle cerebral artery (Fig. 2).

(3) "Absent" if only the ipsilateral middle cerebral o artery, or the ipsilateral middle and anterior cerebral $N$ arteries were visualized (Figs. 3 and 4). 


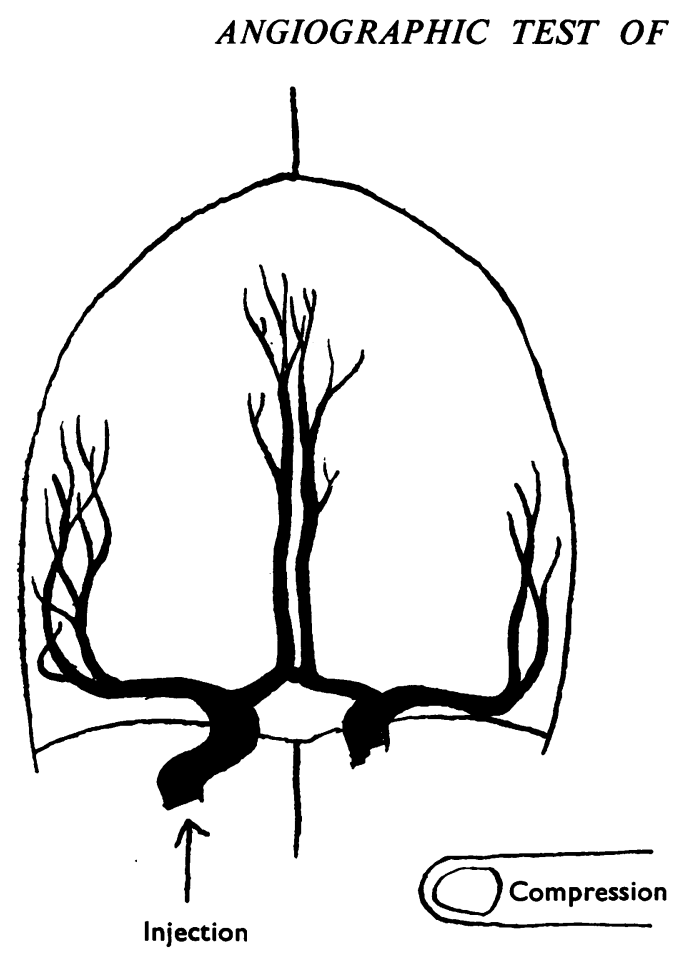

FIG. 1

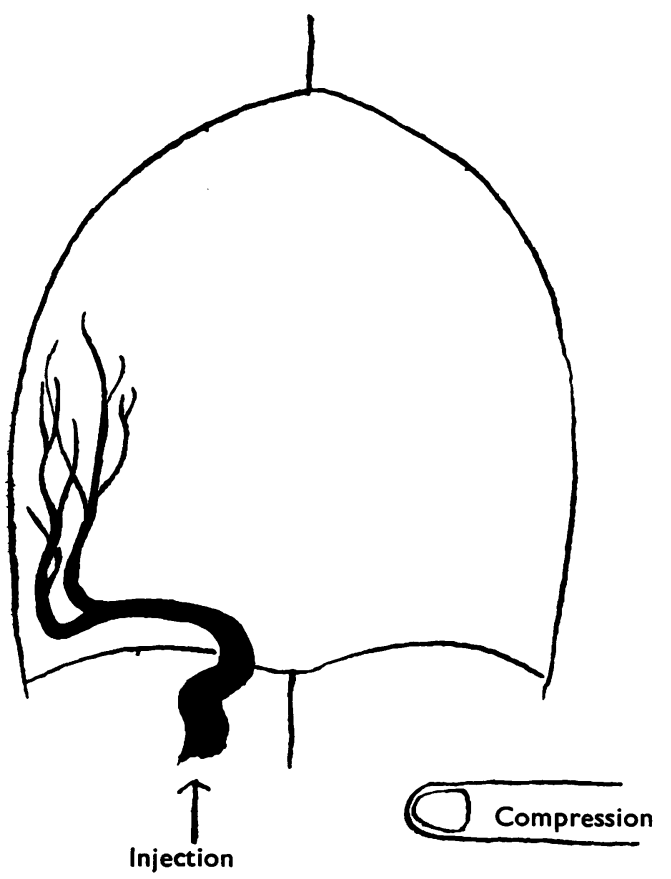

FIG. 3

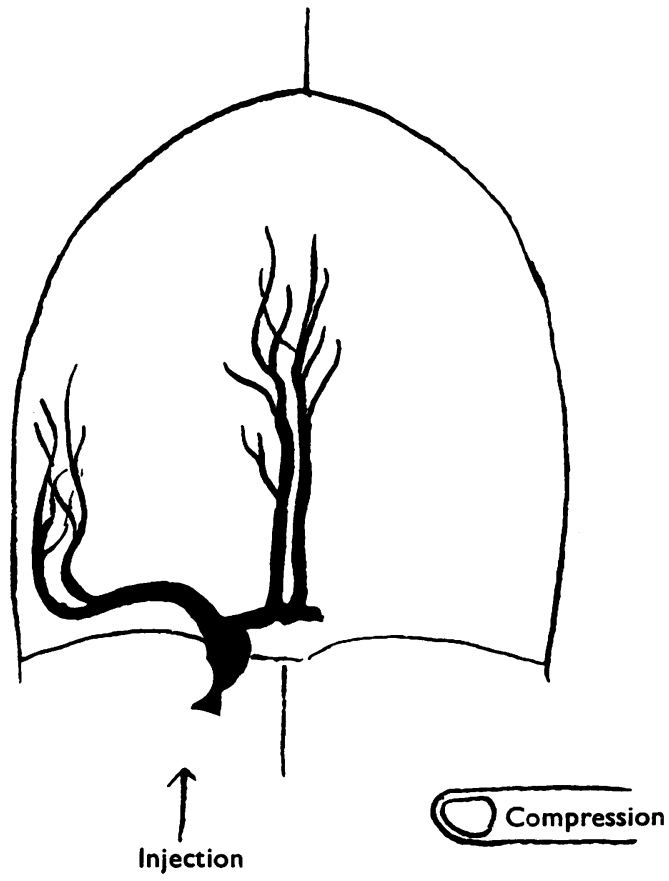

FIG. 2

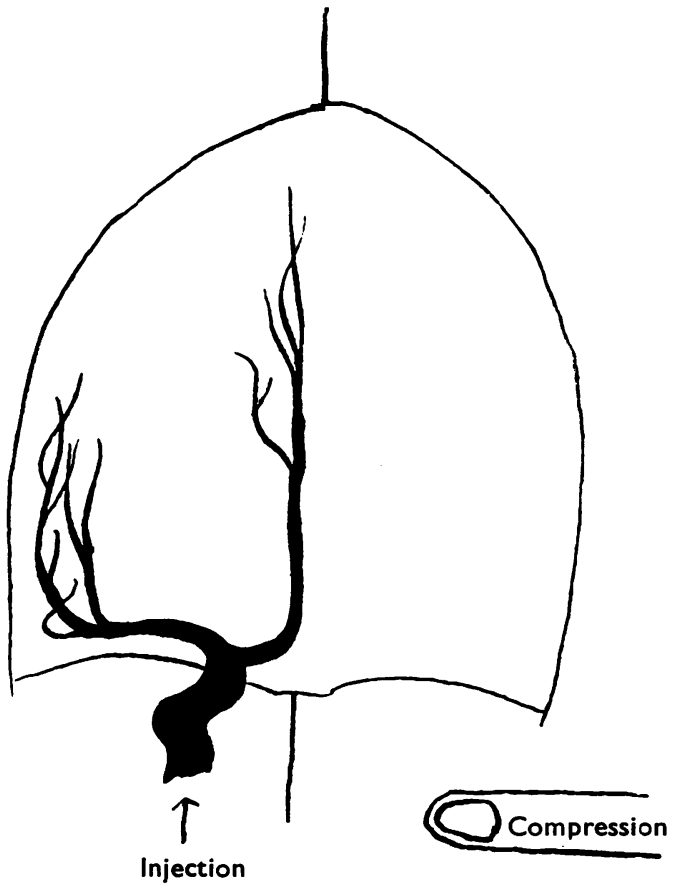

FIG. 4 


\section{Results and Discussion}

In a high proportion of cases of " absent" or " poor" collateral circulations, besides carrying out the test bilaterally, a second and even a third estimation was performed at intervals of a few days to five to six weeks.

Only in 14 patients was the collateral circulation found to be different when tested on separate occasions and only these are included twice in the total number of the tests. For this reason it is stated that in 712 patients the number of collateral circulations tested was 726 , though in fact it was a much larger number.

Of the 726 tests (Table I), in 52 the collateral circulation was "absent" $(7 \cdot 1 \%)$; in 171 it was "poor" $(23.6 \%)$; and in 503 it was "good" $(69 \cdot 3 \%)$.

TABLE I

STATE OF COLLATERAL CIRCULATION IN 712 PATIENTS

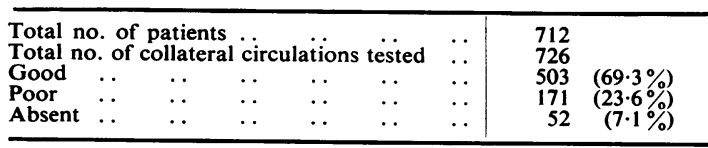

Grouping together all the "vascular conditions" (Table II), there were 364 patients in whom 378 tests were performed. Of these, $31(8.2 \%)$ were defined "absent", $87(23 \%)$ " poor", and 260 $(68.8 \%)$ " good" (Table III). The difference between this group and the overall numbers is trifling.

TABLE II

\section{VASCULAR CONDITIONS}

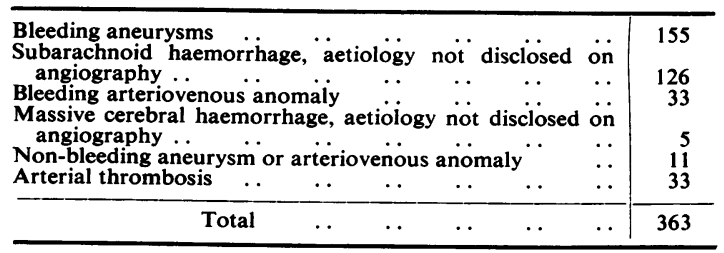

TABLE III

STATE OF COLLATERAL CIRCULATIONS RELATED TO VASCULAR CONDITIONS

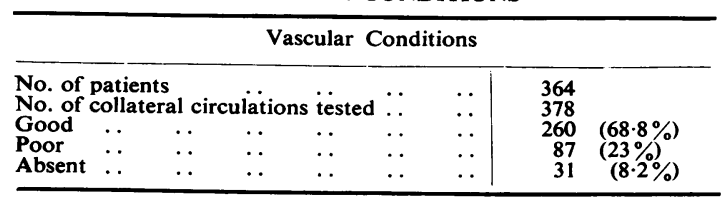

On the other hand it was only in this particular group that in 14 patients the collateral circulation was found different when tested on separate occasions. They constitute approximately $4 \%$ of the vascular cases and $2 \%$ of the overall numbers.
The conditions in which the test was performed in the remaining 348 patients are shown in Table IV. The collateral circulation was found to be absent in $21(6 \%)$, poor in $84(24 \%)$, and good (Table V) in $243(70 \%)$.

TABLE IV

CONDITIONS OF TEST IN REMAINING PATIENTS

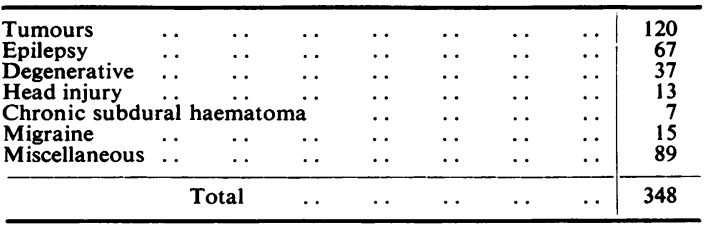

TABLE $\mathrm{V}$

STATE OF COLLATERAL CIRCULATION IN NONVASCULAR CONDITIONS

\begin{tabular}{|c|c|c|c|c|c|c|c|c|}
\hline \multicolumn{9}{|c|}{ Non-vascular Conditions } \\
\hline $\begin{array}{l}\text { No. of } \\
\text { Good } \\
\text { Poor } \\
\text { Absent }\end{array}$ & $\begin{array}{c}\text { coll } \\
\ldots \\
\ldots \\
\ldots\end{array}$ & $\begin{array}{c}\text { al c } \\
\ldots \\
\ldots \\
\ldots\end{array}$ & $\begin{array}{c}\text { Ilati } \\
\ldots \\
\ldots \\
\ldots\end{array}$ & $\begin{array}{l}\text { tested } \\
\ldots \\
\ldots \\
\ldots\end{array}$ & $\begin{array}{l}\ldots \\
\ldots \\
\ldots\end{array}$ & $\begin{array}{l}\cdots \\
\cdots \\
\cdots\end{array}$ & $\begin{array}{r}348 \\
243 \\
84 \\
21\end{array}$ & $\begin{array}{c}(70 \%) \\
(24 \%) \\
(6 \%)\end{array}$ \\
\hline
\end{tabular}

Once again it is evident that there was only añ insignificant difference of the percentages of absene poor, and good collateral circulations between the non-vascular and vascular conditions as well as the overall numbers (cf. Tables I, II, and V).

The analysis of the influence of age on the anatomical channels of collateral circulation acrose the anterior cerebral complex occasioned the greates surprise.

Of the total of 726 tests, 516 fell in the age groups 1-49 years inclusive (the youngest patient was 6 months old). In 37, the collateral circulation was absent $(7.2 \%)$, in 122 was poor $(23.6 \%)$, and in $357(69.2 \%)$ was good. Two hundred and ten tests were performed in the age group $50-70+$ (the oldest patient was 74$)$. In $15(7 \cdot 1 \%)$, it was absent, in $49(23.3 \%)$ it was poor, and in $146(69.6 \%)$ it was good (Table VI).

TABLE VI

STATE OF COLLATERAL CIRCULATION RELATED TO AGE

\begin{tabular}{|c|c|c|c|}
\hline \multicolumn{2}{|c|}{ Age 0-49 } & \multicolumn{2}{|c|}{ Age $50-70+$} \\
\hline $\begin{array}{l}\text { No. of tests } \\
\text { Good } \\
\text { Poor } \\
\text { Absent }\end{array}$ & $\begin{array}{l}516 \\
357(69 \cdot 2 \%) \\
122(23 \cdot 6 \%) \\
37(7.2 \%)\end{array}$ & $\begin{array}{l}\text { No. of tests } \\
\text { Good } \\
\text { Poor } \\
\text { Absent }\end{array}$ & $\begin{array}{l}210 \\
146(69.6 \%) \\
49(23.3 \%) \\
15(7 \cdot 1 \%)\end{array}$ \\
\hline
\end{tabular}

Evidently there is no real difference between the collateral circulation across the anterior segment of the circle of Willis in the under-50s and the over50s. These percentages are, in turn, practically o identical with the findings obtained by analysis of $N$ the aetiological groups. 
It was thought unimportant to work out statistics for subdivisions of the aetiological conditions enumerated in Tables II and IV or to subdivide the ages into decades. The small numbers of tests in each such subdivision would make any statistical analysis impracticable.

Poor collateral circulation was found in this series to be in the order of 23 to $24 \%$, irrespective of age or pathology. Compression of the opposite internal carotid artery was digital and percutaneous. It is, therefore, conceivable that a proportion of this percentage was the result of an inefficient compression. Thus, what may have been cases of good collateral circulation became poor, according to the adopted definition, and this may have occurred in $\pm 10 \%$ of tests. In another, as yet indefinite percentage, the primary source of collateral circulation was presumably through the posterior segment of the circle of Willis. A full experimental study is still needed here.

Finally, some cases classified in this group were due to the dynamic influences which are less generally appreciated but have been discussed by some authors (Sweet and Bennett, 1948; Shenkin, Harmel, and Kety, 1948; Shenkin, Cabieses, van den Noordt, Sayers, and Copperman, 1951; Stern, 1953; Strobos and Mount, 1953; Brown, 1955).

The circle of arterial channels at the base of the brain known to us as the circle of Willis was recently the subject of an admirable review by Sir Charles Symonds (1955). The anterior segment of this circle comprises the supraclinoid segment of the two internal carotid arteries, proximal segments of the anterior cerebral arteries which are joined together by the anterior communicating artery. This arterial complex may be defective as a result of a congenital abnormality. It may take the form of an absent, or grossly underdeveloped anterior communicating artery or a similar condition involving the proximal segment of one anterior cerebral artery. When this last anomaly is present the arteriographic appearance or the post-mortem anatomical dissection shows the two anterior cerebral arteries as an integral system of one internal carotid artery. The other internal carotid artery has as its main branch the middle cerebral artery. It then takes no part in the formation of the anterior segment of the circle of Willis. The collateral circulation in such cases is absent, but the angiographic test must be performed bilaterally and repeated at a second investigation. When these criteria were fulfilled only a very few cases of such an anomaly were proved, and usually though not exclusively in the presence of an aneurysm of the anterior cerebral arterial complex. This combination is well known although sometimes overemphasized.
As stated previously, in the groups of patients with vascular pathology there were 14 out of 364 in whom different results were obtained when the test was performed on separate occasions (4\%). The reason for the alterations was segmental spasm affecting the part of the circle under discussion. All these were cases of intracranial bleeding from a verified aneurysm subjected to angiography from a few hours to a few days after the haemorrhage. The test was repeated at intervals varying from a few days to three weeks. In each of these patients an absent or poor collateral circulation became poor or good accordingly. Although the patients with arterial anomalies and arterial spasm represent only a small percentage of the vascular cases, they must be taken into careful consideration when contemplating surgical treatment of intracranial haemorrhage. It was felt important to draw attention to these cases. Moreover, having done so, it is reemphasized that absent collateral circulation was found in 31 patients $(8.2 \%)$ in this group. In the groups comprising all other pathological conditions, there were $21(6 \%)$ patients out of 348 . The difference of $2.2 \%$ is of no great significance in a series of this size.

The limitations of this angiographic experiment must be acknowledged, since it can never be an absolutely true image of the circulation. The lack of an apparatus in this Centre which would allow taking more than three exposures is highly regretted. Despite these reservations, it is felt that the most interesting finding in this series is the apparent refutal of the common belief that age materially affects the collateral circulation through the circle of Willis. It may be that in younger age groups bleeding conditions and anatomical anomalies play a greater part, whereas in the older groups dynamic influences predominate as factors affecting the collateral circulation. It is also true that there were 516 patients in the group of under 50 years of age and only 210 in the group over 50 . Nevertheless, in the former group collateral circulation was absent $7.2 \%$ (37 times), poor $23.6 \%$ (122 times), while in the latter it was absent in $7 \cdot 1 \%$ (15 times) and poor in $23.3 \%$ (49 times) (Table VI). These percentages are identical and it is felt that they cannot be disregarded.

Under the conditions of this experiment, the anterior segment of the circle of Willis seemed anatomically and dynamically an admirable device for the carriage of the blood supply in health and its redistribution in stress.

\section{Summary}

An angiographic test estimating the collateral circulation through the anterior segment of the 
circle of Willis has been carried out in 712 patients.

The results were analysed in relation to age and pathology.

\section{REFERENCES}

Brown, A. S. (1955). Anaesthesia, 10, 346.

Sedzimir, C. B. (1955). J. Neurosurg., 12, 460.
Shenkin, H. A., Harmel, M. H., and Kety, S. S. (1948). Arch. Neurol. Psychiat. (Chicago), 60, 240.

Cabieses, F., Noordt, G. van den, Sayers, P., and Copperman, R. (1951). J. Neurosurg., 8, 38 .

Stern, W. E. (1953). Ibid., 10, 577

Strobos, R. R. J., and Mount, L. A. (1953). A.M.A. Arch. Neurol. Psychiat., 69, 118.

Sweet, W. H., and Bennett, H. S. (1948). J. Neurosurg., 5, 178. Symonds, Sir Charles (1955). Brit. med. $\mathcal{X}$. 1, 119.

\section{THE NOVEMBER (1958) ISSUE}

The November (1958) issue contains the following papers:-

Further Observations on Subarachnoid Haemorrhage. Wylie McKissock, Kenneth Paine, and Lawrence

Walsh

Leucotomy for Pain. Alick Elithorn, Eric Glithero, and Eliot Slater

A Controlled Study of the Effects of Leucotomy. A. A. Robin

Thyrotoxic Myopathy. Ragnar Hed, Lennart Kirstein, and Curt Lundmark

Individual Variation in the Size of the Tentorial Opening. J. A. N. Corsellis

Arteries of the Spinal Cord in the Cat. Peter Bradshaw

Accommodation in the Holmes-Adie Syndrome. G. F. M. Russell

The Effect of Reserpine on the Duration of Manic Attacks. David C. Watt

A Controlled Trial of Meprobamate in Anxious Out-Patients. John M. Hinton

Myeloid Cells in Cerebrospinal Fluid. A. I. Spriggs

Two Hundred and One Cases of Intracranial Tuberculoma Treated Surgically. C. Arseni

Book Reviews

Index to Volume 21

A number of copies are still available and may be obtained from the Publishing Manager, British Medical Association, Tavistock Square, W.C.1, price 17s. $6 \mathrm{~d}$. 